\title{
Erfolge und Herausforderungen im Kampf gegen Infektionskrankheiten
}

\author{
N. J. Mueller ${ }^{a}$, B. Hasse ${ }^{b}$, für die Schweizerische Gesellschaft für Infektiologie \\ a Präsident, Schweizerische Gesellschaft für Infektiologie \\ b Wissenschaftliche Sekretärin, Schweizerische Gesellschaft für Infektiologie
}

Die wohl spektakulärste Innovation der Infektiologie in den letzten 20 Jahren war die Entwicklung einer Therapie für HIV, die aus einer tödlichen eine behandelbare Erkrankung gemacht hat - mit einer fast normalen Lebenserwartung und Lebensqualität für die Betroffenen. Grossen Nutzen brachten auch neue Behandlungen von Hepatitis C, Impfungen und Antibiotika. Gefahren durch Epidemien und zunehmende Resistenzen fordern uns aber weiterhin heraus.

Aktuell leben zwischen 13000 und 20000 Menschen in der Schweiz mit HIV. Die Erfolge in der Behandlung von HIV haben das Gesicht dieser Krankheit fundamental geändert. HIV ist eine in der Mehrzahl der Fälle gut kontrollierbare, chronische Krankheit geworden. Bei werdenden Müttern mit HIV-Infektion kann heute durch eine konsequente Behandlung eine Übertragung auf das Kind fast in jedem Fall verhindert werden. Die opportunistischen Erkrankungen werden deutlich weniger häufig beobachtet, und internistische Probleme stehen heute bei vielen HIV-Patienten im Vordergrund. Mit einer oder zwei Kombinationstabletten pro Tag kann die HIV-Replikation bei den meisten Infizierten unterdrückt werden, so dass die so Behandelten das Virus nicht mehr weitergeben können. Erhielten alle Menschen weltweit eine solche Behandlung, könnte HIV sogar eliminiert werden. Aktuelle Programme der UNAIDS zielen denn auch darauf hin, diese Behandlungen auch in ressourcenarmen Ländern einzusetzen. So sollten 90\% der Menschen mit HIV von ihrer Infektion wissen, $90 \%$ davon Medikamente erhalten und bei $90 \%$ davon sollte die Plasma-HIV-RNA nicht mehr nachweisbar sein, so dass sie nicht mehr infektiös sind.

\section{Nicht nur HIV, auch Hepatitis C ist heute behandelbar}

An Hepatitis Cleiden in der Schweiz gemäss einer Studie der Universität Bern 0.7\% der Bevölkerung, wovon noch etwa 43000 unbehandelt sind. Während die Hepatitis C noch vor wenigen Jahren eine mehrheitlich chronisch verlaufende Erkrankung war, kann sie heute mit den sogenannten "directly acting antivirals» (DAA) in der überwiegenden Mehrzahl der Fälle anhaltend geheilt werden. Damit kann auch das Fortschreiten der Erkrankung zur Leberzirrhose und dem gefürchteten Leberzellkarzinom verhindert werden. So sparen diese kurzfristig zwar teuren - Behandlungen letztlich Geld ein, da die kostenintensiven Behandlungen der andernfalls entstehenden Folgeerkrankungen entfallen.

\section{Impfungen: fortgesetzte Erfolgsgeschichte}

Impfungen gelten als Paradebeispiel für das Konzept der Prävention und haben sich millionenfach bewährt. Neben den etablierten Impfungen kam 2006 eine Impfung mit einem ganz neuen Konzept auf den Markt: Die Impfung gegen humane Papillomaviren (HPV) dient nicht primär der Verhinderung der von ihnen unmittelbar ausgelösten Erkrankung, welche in der Regel asymptomatisch ist, sondern sie verhindert die Spätfolgen, nämlich die genitalen und oralen Karzinome. Es besteht begründete Hoffnung, dass die Häufigkeit von Gebärmutterhalskrebs entscheidend zurückgeht. Dies wie auch die Therapien für HIV und HPV nutzt nicht nur individuellen Patienten, sondern auch der Volkswirtschaft: Erkrankungen oder Komplikationen werden verhindert, Patienten geheilt und ein uneingeschränktes Erwerbsleben ermöglicht.

\section{Antibiotika: ein Pfeiler der Prävention und Behandlung}

Antibiotika haben in den letzten 70 Jahren wesentlich zur längeren Lebenserwartung beigetragen und sind aus dem medizinischen Alltag nicht mehr wegzudenken. Neben ihrem Einsatz in der Primärversorgung sind sie für den Langzeiterfolg von komplexen Be- 
handlungen wie z.B. Transplantationen, aplasierenden Chemotherapien oder Polytraumata unabdingbare Voraussetzung.

Die antibiotische Prophylaxe vor, während und nach Operationen verringert signifikant die Häufigkeit von Infektionen insbesondere nach Operationen mit Fremdmaterialimplantaten wie Herzschrittmachern, Gelenk- oder Gefässprothesen sowie in der Viszeralchirurgie. Der zeitlich korrekte Einsatz des vom Spektrum her richtigen Antibiotikums ist hier für den Erfolg entscheidend.

Infektiologinnen und Infektiologen stehen im täglichen Einsatz in der Praxis und im Spital dafür ein, dass diese so wertvollen Substanzen in Prophylaxe und Therapie gezielt eingesetzt werden und den wahrscheinlichen Erreger im Spektrum haben. Aufgrund der richtigen raschen mikrobiologischen Diagnostik muss das Antibiotikum gezielt deeskaliert und nur so lange wie nötig verabreicht werden. Damit werden der Gebrauch von Antibiotika und damit auch der Antibiotikaresistenzdruck insgesamt reduziert.

\section{Die riesige Herausforderung der zuneh- menden Resistenzen von Antibiotika}

Um den ausgezeichneten Standard der medizinischen Versorgung in der Schweiz zu erhalten, benötigen wir gut wirksame Antibiotika. Die Entwicklung neuer und besserer Antibiotika oder alternativer Methoden war in den letzten Jahren keine Priorität mehr, obwohl sich durch den hohen Antibiotikakonsum weltweit antibiotikaresistente Bakterien verbreiteten. Dies hat zu einer kritischen Versorgungslücke geführt. Mit der nationalen Strategie gegen Antibiotikaresistenzen (StAR) soll durch Interventionen in der Landwirtschaft, in der Human- und Veterinärmedizin diese kritische Phase überwunden werden. Die Infektiologie/Spitalhygiene ist in diesen Prozess intensiv involviert. Auf allen Stufen muss das Bewusstsein geschärft werden, dass nur durch einen sehr bewussten und gezielten Gebrauch der Antibiotika eine noch grössere Krise verhindert werden kann: Der wachsende Nutzen der Medizin, auch ausserhalb der Infektiologie, wird in vielen Gebieten nur dann nachhaltig sein, wenn wir auch in Zukunft über wirksame Antibiotika verfügen.

Antibiotikaresistenz ist und war nie ein isoliertes Problem, sondern breitet sich epidemisch weltweit aus. Auch wenn in der Schweiz noch vorwiegend die stationäre Medizin betroffen ist, ist auch der Arzt in der Praxis zunehmend mit dieser Herausforderung konfrontiert. Noch vor wenigen Jahren war z.B. die Abkürzung ESBL (Extended Spectrum Beta Lactamase) ein vorwiegend dem Spezialisten bekanntes Phänomen. Heute ist dieser
Resistenzmechanismus auch dem Praktiker geläufig und erschwert die ambulante Behandlung erheblich. Die Infektiologie/Spitalhygiene leistet hier einen über die individuelle Medizin hinausgehenden Beitrag, ist sie doch dafür besorgt, dass Antibiotika rational eingesetzt werden und es insbesondere in Spitälern zu keiner Übertragung von resistenten Erregern von Patient zu Patient kommt. Viele Aspekte von der Veterinärmedizin bis zum persönlichen Gebrauch müssen in eine Gesamtstrategie einfliessen: Der Ökonomieprofessor Jim O’Neill sagt voraus, dass bis 2050 mehr Menschen an wegen Antibiotikaresistenzen nicht mehr behandelbaren Infektionskrankheiten sterben als an Krebs oder kardiovaskulären Erkrankungen. Es geht uns alle etwas an!

\section{Epidemien in einer vernetzten Welt}

Neben den Antibiotikaresistenzen sind infektiöse Epidemien eine möglicherweise unterschätzte Gefahr. In unserer immer besser vernetzten Welt verteilen sich Erreger und Infizierte effizient rund um den Globus. Durch die globale Erwärmung finden sich Insekten, die für die Übertragung von Infektionen verantwortlich sind, auch in gemässigteren Klimazonen. Eine lokale Epidemie kann sich dadurch rasch ausbreiten, und Erkrankte müssen überall erwartet werden. Neu besteht die Gefahr, dass gewisse Erreger auch bei uns heimisch werden. Die Vorbereitung und Planung für solche Ausnahmesituationen sind äusserst zeit- und ressourcenintensiv. Der Schutz der Bevölkerung hat hier oberste Priorität. Leider ist jedoch der unmittelbare Nutzen von präventiven Massnahmen häufig schwierig zu vermitteln, solange die Katastrophe nicht eingetreten ist.

\section{Fazit}

Der individuelle Nutzen der Infektiologie durch moderne Behandlungskonzepte ist offensichtlich. Weniger im Vordergrund ist die wichtige Aufgabe im Bereich der Prävention: Infektiologinnen und Infektiologen widmen sich in der Gruppe «Swissnoso» ganz gezielt der Prävention von Spitalinfektionen. Zudem unterstützen sie andere Ärzte mit Guidelines (www.ssi.guidelines.ch) in therapeutischen Entscheidungen bei Infektionskrankheiten und Präventionsmassnahmen (www. swissnoso.ch). Die Schweizerische Gesellschaft für Infektiologie arbeitet auch sehr eng mit den Kantonen und dem Bundesamt für Gesundheit (BAG) zusammen, da Epidemien nicht an den Kantons- und Landesgrenzen haltmachen. Nur ein optimales Zusammenspiel von wirksamer Infektprävention und -behandlung sichert langfristig die Erfolge von der Primärversorgung bis zur komplexen tertiären Medizin. 